
anerythrus)

\title{
3 Materials and Methods
}

4 DNA was extracted from 100-200 ul of whole blood from each rope squirrel. 400 base pairs of

5 the mitochondrial cytochrome b (Cyt b) gene was amplified and sequenced. Primers were 5'

6 CGA AGC TTG ATA TGA AAA ACC ATC GTT G 3’ (MVZ05) and 5’ GCC CTC AGA

7 AGG ATA TTG TCC TCA TGG 3’ (400R) [39]. PCR products were purified using Zymo PCR

8 purification kit (Zymo Research, Irvine, CA) and the amplication concentration was calculated

9 using a Nanodrop 2000 spectrophotometer (Nanodrop, Wilmington, DE). Sanger sequencing

10 was performed at the University of Wisconsin Biotechnology Center (Madison, WI). DNA

11 sequences were edited and aligned using Geneious 8.0.5 software. Phylogenetic trees were

12 constructed using a neighbor-joining algorithm (Kimmura and 1,000 bootstraps) in MEGAN5

13 software [40]. BLAST search in non-redundant databases were performed and sequences were

14 submitted to NCBI GeneBank. The GeneBank AY371098.1 and AY371103.1 were used as

15 outgroups in phylogenetic analyses.

\section{Results and Discussion}

17 In the BLAST search, no rope squirrel (Funisciurus sp.) matching sequences were encountered.

18 The closest related sequences belonged to the particolored flying squirrel (Hylopetes alboniger).

19 The phylogenetic reconstruction showed that two main branches were supported (bootstraps =

20 100) (Fig. A). The experimental infection groups were distributed between the two branches.

21 These are the first sequences reported in the genus Funisciurus. The distance of the branches

22 and number of nucleotide substitutions suggests that the study population displayed high genetic 
23 diversity. This may have impacted the diversity of pathological outcomes found in the infection

24 trial. However, morbidity and mortality occurred within both genetic groups.

25

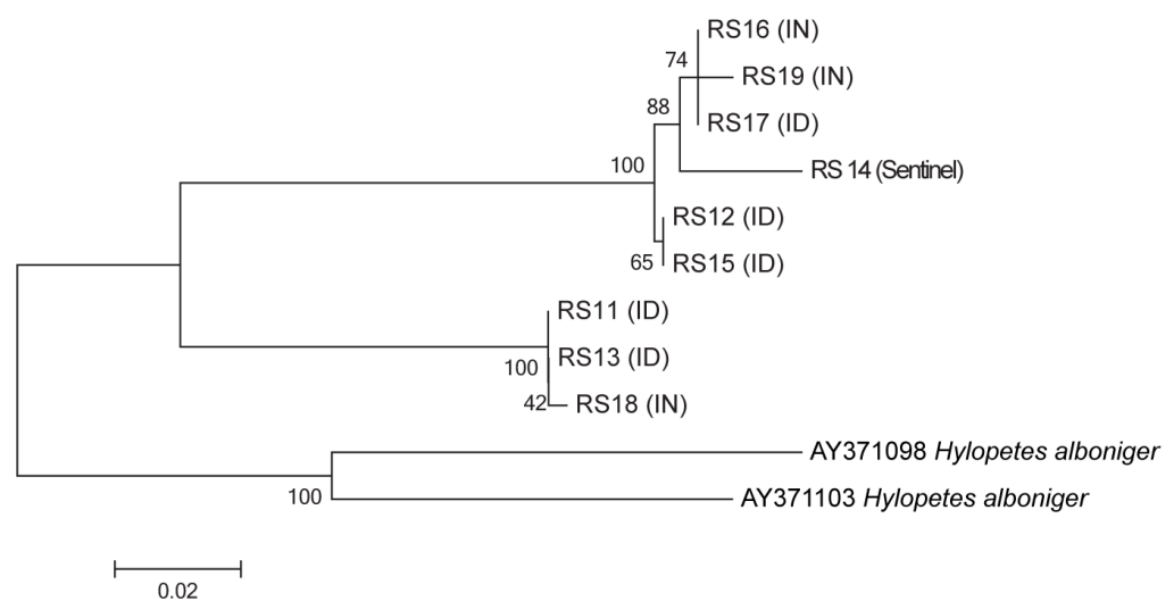

26

27 Figure A. The phylogenetic tree, constructed based on 400 bp sequences of the cytochrome b

28 gene, show that there are two different genetic groups within the group of rope squirrels,

29 (Funisciurus anerythrus) infected with Monkeypox virus. The infection groups are indicated

30 after the individual animal numbers, as either intradermal (ID), intranasal (IN), or sentinel. 